\title{
Delivery of ROS Generating Anthraquinones Using Reduction-responsive Peptide-based Nanoparticles
}

\author{
Pascal U. Richard, ${ }^{a}$ Ioana Craciun, ${ }^{a}$ Jens Gaitzsch, ${ }^{a}$ Lev Weiner, ${ }^{b}$ Cornelia G. Palivan ${ }^{\star}, a$ \\ ${ }^{a}$ Department of Chemistry, University of Basel, Mattenstrasse 24a, CH-4058 Basel, Switzerland, E-mail: cornelia.palivan@unibas.ch (C.P)

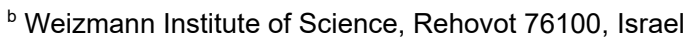

\begin{abstract}
In order to limit the side effects associated with antitumor drugs such as doxorubicin, nanosized drug-delivery systems capable of selectively delivering and releasing the drug in the diseased tissue are required. We describe nanoparticles (NPs), self-assembled from a reduction responsive amphiphilic peptide, capable of entrapping high amounts of a redox active anticancer drug candidate and releasing it in presence of a reducing agent. This system shows a high entrapment efficiency with up to $15 \mathrm{mg}$ drug per gram of peptide (5.8 mol\%). Treatment of the NPs with reducing agent results in the disassembly of the NPs and release of the drug molecules. A reduction in cell viability is observed at drug concentrations above $250 \mathrm{nM}$ in HEK293T and HeLa cell lines. This drug delivery system has potential for targeting tumor sites via the EPR effect while taking advantage of the increased reduction potential in tumor microenvironment.
\end{abstract}

Keywords: Peptide • Self-assembly • Drug Delivery • Quinone • Multicompartment Micelles

\section{Introduction}

While cancer cases keep increasing, the major pharmaceutical treatments for tumors rely on chemotherapy, which results in numerous side effects. One of the major chemotherapeutic compounds is the anthracycline doxorubicin (DOX) which is known for its beneficial therapeutic effects on multiple forms of cancer, but also causes serious side effects, notably significant cardiotoxicity. ${ }^{[1,2]}$ One approach to reduce such side effects is through the use of nanosized drug delivery systems (DDS). Classic DDS are either composed of inorganic materials or made from self-assembled soft matter ${ }^{[3-5]}$ The latter are typically composed of amphiphilic molecules that can be loaded with various molecules of therapeutic interest. ${ }^{[6-9]}$ The use of nanosized DDS allows for increased circulation times and nanoparticles of sizes up to $200 \mathrm{~nm}$ are known to accumulate in tumor tissue through the enhanced permeability and retention (EPR) effect, ${ }^{[6,}$ 10] thus allowing passive targeting of tumor sites. Already used clinically, liposomal formulations of DOX have been shown to moderately reduce the cardiotoxic side effects associated with administering the free drug. ${ }^{[1,2,6]}$

Numerous biocompatible amphiphiles (e.g. lipids, diblock and triblock copolymers, peptides) have been shown to form nanoparticles (NPs) of different morphologies, such as micelles, vesicles and other complex structures. ${ }^{[7-9,}{ }^{11]}$ These nanostructures differ in size and ability to be loaded with various compounds. Structures containing a large aqueous lumen such as vesicles, for example, are favored for the delivery of hydrophilic drugs, whereas micelles are usually more suited for the delivery of hydrophobic drugs. ${ }^{[6-9]}$ More complex selfassembly structures, namely multi-compartment-micelles (MCMs) can host both hydrophilic and hydrophobic drugs, making them especially versatile candidates for drug delivery. We have recently reported the redox-responsive amphiphilic peptide $\mathrm{H}_{3} \mathrm{SSgT}$ (of the sequence $\mathrm{H}_{2} \mathrm{~N}$ -
$\mathrm{H}_{3}-\mathrm{X}-[\mathrm{W}-\mathrm{D} \mathrm{L}]_{3}-\mathrm{W}-\mathrm{CONH} \mathrm{H}_{2}$, where $\mathrm{X}=-\left(\mathrm{CH}_{2}\right)_{2}-\mathrm{S}-\mathrm{S}-\left(\mathrm{CH}_{2}\right)_{2}-\mathrm{NH}-\mathrm{CO}-\left(\mathrm{CH}_{2}\right)_{2}-\mathrm{CO}-$ and $D L=D$-Leucine (Figure 1), that forms such MCMs. ${ }^{[12-14]}$ They were shown to efficiently entrap the model compound borondipyrromethene (Bodipy) and release it in presence of physiological amounts of reducing agent. ${ }^{[13]}$ The MCMs are about $150 \mathrm{~nm}$ in diameter, making them suitable to deploy the EPR effect for tumor tissue uptake. In addition to a leaky vasculature leading to the EPR effect, the environment surrounding tumor tissues possess abnormal physico-chemical properties such as decreased $\mathrm{pH}$, increased temperature and unusual redox potentials. ${ }^{[15-17]}$ Due to the redoxresponsive nature of the peptide used to form the MCMs, the system also has a built-in stimuli responsive delivery mechanism. ${ }^{[16,18-20]}$ We now aim towards taking this system a step further and apply it for a drug rather than model compounds.

With respect to the target drug molecule, we focused on current research aimed at developing compounds with higher potency than DOX. One example of such a class of compounds are the anthraquinones with metal chelating abilities inspired from anthracyclines. While the exact mechanism of anthracycline-induced cytotoxicity is still unknown, the production of highly reactive free radicals and reactive oxygen species (ROS) is a generally accepted mechanism. ${ }^{[2,6,21]}$ It arises from the presence of a quinone moiety that can be readily reduced by cellular reductants to yield a semiquinone radical. ${ }^{[21]}$ In aerobic conditions, semiquinones can reduce molecular oxygen resulting in the formation of ROS, which can initiate radical chain reactions leading to oxidative stress and ultimately cellular death. ${ }^{[6]}$ Given the importance of redox-active metal ions in the mechanism of toxicity of anthracyclines, it has been suggested that metal-chelating quinone moieties could considerably increase the rate of ROS generation, thus leading to higher cytotoxicity. $\left.{ }^{[21,} 22\right]$ Anthraquinone derivatives with chelating groups were shown to generate higher ROS levels than DOX in vitro, ${ }^{[21,23,24]}$ making them 
interesting candidates for cancer therapy, however, their cellular cytotoxicity has not been reported so far.
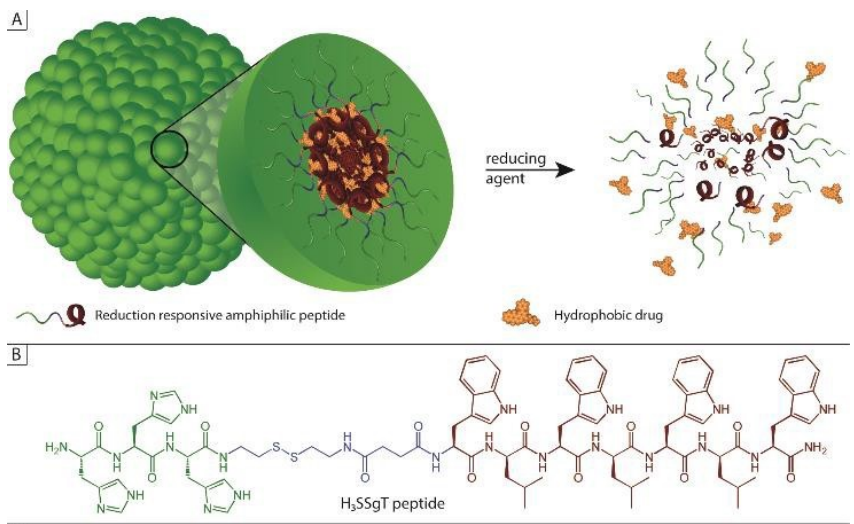

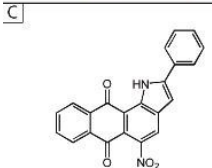

Qn

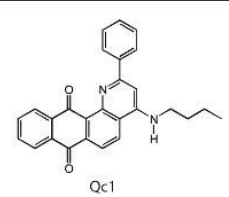

Qc1

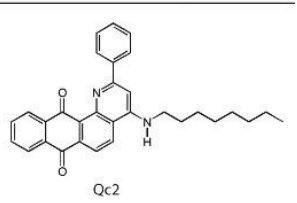

Qc2
Figure 1. A) Schematic representation of anthraquinone loaded peptide NPs and their disassembly in presence of reducing agent. B) Structure of the $\mathrm{H}_{3} \mathrm{SSgT}$ peptide. The hydrophilic tri-histidine (green) is linked to the hydrophobic $[\mathrm{W}-D \mathrm{~L}]_{3}$ sequence (red) through a disulfide containing linker (blue). C) Structure of the anthraquinone derivatives $\mathrm{Qc1}, \mathrm{Qc2}$ and $\mathrm{Qn}$.

Using our MCMs, we entrapped three different anthraquinone derivatives as anticancer drug candidates (Qn, Qc1 and Qc2) (Figure $1 \mathrm{C})$, and evaluated the entrapment efficiency, morphology and size of the loaded NPs. Cytotoxicity of the compound with the highest loading efficiency, Qc2, was assessed on both HEK293T and HeLa cells. Finally, we confirmed the entrapment of Qc2 in the peptide NPs as well as release under reductive conditions using both cell lines. Our research aims at developing this stimuli-responsive NP-drug system into a possible alternative to currently used chemotherapies.

\section{Results and Discussion}

\section{Nanoparticles Formation and Characterization}

The amphiphilic peptide $\mathrm{H}_{3} \mathrm{SSgT}$ self-assembles into MCMs, obtained from the aggregation of individual micelles (Figure 1A). These particles are formed by solvent exchange of ethanolic solutions to purified water $\left(\mathrm{ddH}_{2} \mathrm{O}\right)$, with multiple parameters such as the concentration of the peptide and the solvent exchange rate affecting their final size and morphology. In the case of the peptide $\mathrm{H}_{3} \mathrm{SSgT}$, dialysis of a $0.2 \mathrm{mg} \mathrm{mL}^{-1}$ solution of the peptide in $50 \%$ ethanol against water allowed for the formation of particles of desired size and morphology. Imaging of the obtained NPs by transmission electron microscopy (TEM), revealed spherical structures (Figure 2 and S1) and dynamic light scattering (DLS) measurements showed that the NPs exhibited a mean hydrodynamic diameter $\left(D_{H}\right)$ between 200 and $300 \mathrm{~nm}$ (Table 1 and S1). Transfer of the NP suspension into 3-(Nmorpholino)propanesulfonic acid (MOPS, pH 6.5) buffer resulted in a shrinking of the particles without otherwise affecting their morphology, as observed by TEM (Figure S2) and DLS (Table S1). The observed shrinking of the NPs can be attributed to the increased osmotic pressure resulting from the buffer. After their preparation in deionized water, the nanoparticles were transferred into MOPS buffer, which considerably increased the salt concentration and induced the mentioned osmotic pressure. Since this system is designed for passive targeting using the EPR effect, smaller sizes are advantageous.
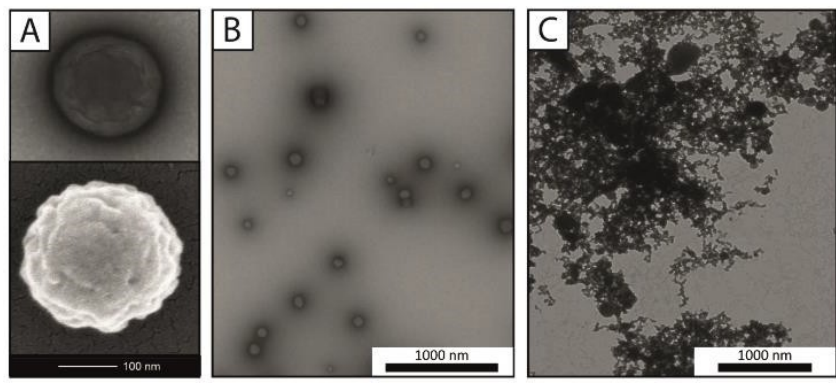

Figure 2. A) Individual peptide NP $s$ as observed by transmission (top) and scanning (bottom) electron microscopy. Scale bar $=100 \mathrm{~nm}$. B) TEM micrograph of representative samples of Qc2-loaded peptide NPs and C) TEM micrograph of Qc2-loaded NPs after incubation (2h) with reducing agent TCEP.

\section{Payload Embedding and Characterization of Loaded}

\section{Nanoparticles}

Entrapment of three different redox active anticancer drug candidates Qn, Qc1 and Qc2 (Figure 1C) was evaluated upon self-assembly of the peptide NPs. All three anthraquinone derivatives possess similar redox potentials, but differ with respect to their hydrophobicity, with Qc2 being the most hydrophobic due to its longer aliphatic side chain. Qc2 has an octyl side chain, Qc1 possesses only a butyl chain and Qn bares no side chain at all. As the peptide NPs are MCMs, they possess multiple hydrophobic inner cores and thus preferentially entrap hydrophobic compounds. ${ }^{[13,14]}$ Therefore, the structural differences between these anthraquinones can affect their loading efficiency within the hydrophobic cores of the peptide NPs. To prepare the loaded NPs, the anthraquinone was added to a peptide solution, diluted to a final concentration of $6-12 \mu \mathrm{M}$ in $50 \%$ ethanol and dialyzed against water. The presence of the anthraquinones did not significantly affect the shape and size of the particles (Table 1, Figure S3).

The concentration of anthraquinones in the NP suspension was quantified by reversed phase high-performance liquid chromatography (RP-HPLC) (Figures S7 and S8). The resulting concentrations were determined to be $2.8 \pm 0.8 \mu \mathrm{M}$ for $\mathrm{Qn}, 3.8 \pm 0.7$ $\mu \mathrm{M}$ for Qc1 and $6.6 \pm 0.6 \mu \mathrm{M}$ for Qc2, in the NPs formed from initial concentrations of $0.2 \mathrm{mg} \mathrm{mL}^{-1}$ peptide and $12 \mu \mathrm{M}$ of the anthraquinones (Table 1). In order to verify that the anthraquinones are indeed entrapped within the peptide NPs and not free in solution, a $12 \mu \mathrm{M}$ solution of the free anthraquinone Qc2 was dialyzed following the same procedure as for NP formation. After dialysis, the detectable concentration was below $0.8 \mu \mathrm{M}(<6 \%$ initial concentration) (Figure S9), thus confirming efficient removal of the non-entrapped compounds. This confirms that, upon peptide NP formation, the anthraquinones are successfully entrapped within the particles and not free in solution. 
The concentrations of entrapped anthraquinones Qc1 and Qn $(2.8 \pm$ $0.8 \mu \mathrm{M}$ and $3.8 \pm 0.7 \mu \mathrm{M}$ ) are in line with the $3 \mu \mathrm{M}$ previously reported for the hydrophobic model compound Bodipy ${ }^{[13]}$ and correspond to loadings of $5 \mathrm{mg}$ anthraquinone per gram of peptide (2.4 $\pm 0.4 \mathrm{~mol} \%)$ for Qn and $8 \mathrm{mg} \mathrm{g}^{-1}$ (3.3 $\left.\pm 0.6 \mathrm{~mol} \%\right)$ for Qc1. In the case of Qc2, the loading efficiency was two times higher, reaching concentrations up to $7 \mu \mathrm{M}$, corresponding to $15 \mathrm{mg} \mathrm{Qc2}$ per gram of peptide (5.8 \pm 0.5 $\mathrm{mol} \%$ ) (Table 1 and S1). The higher entrapment efficiency of this compound, compared to Qc1, is due to the presence of a longer aliphatic chain (octyl for Qc2, butyl for Qc1), thus increasing its hydrophobicity. As a result, higher entropic driving forces are present between Qc2 and the hydrophobic cores of the NPs. The high loading efficiency for compound Qc2 supports its case as a candidate for cancer therapy and was selected for further evaluation.

Table 1. Loading efficiency and sizes of peptide NPs loaded with the anthraquinone derivatives Qn, Qc1 and Qc2.

\begin{tabular}{|c|c|c|c|c|c|}
\hline \multirow{2}{*}{$\begin{array}{l}\text { Pay- } \\
\text { load }\end{array}$} & \multicolumn{3}{|c|}{ Anthraquinone loading } & \multirow{2}{*}{$\begin{array}{l}\begin{array}{r}\text { Size in } \\
\text { ddH2O }\end{array} \\
\text { Rh [nm] } \\
\text { (PDI) }\end{array}$} & \multirow{2}{*}{$\begin{array}{l}\text { Size in MOPS } \\
\quad \mathrm{pH}=6.5 \\
\mathrm{Rh}[\mathrm{nm}] \\
\text { (PDI) }\end{array}$} \\
\hline & $\begin{array}{l}\mathbf{c t}^{t} \\
{[\mu \mathrm{M}]}\end{array}$ & $\begin{array}{l}\text { molar } \\
\text { ratio [\%] }\end{array}$ & $\begin{array}{l}\text { [mg g-1 } \\
\text { peptide] }\end{array}$ & & \\
\hline none & & & & $137(0.30)$ & $\mathrm{N} / \mathrm{A}$ \\
\hline Qn & $\begin{array}{l}2.8 \pm \\
0.8\end{array}$ & $2.4 \pm 0.4$ & $5 \pm 1$ & $128(0.36)$ & $\mathrm{N} / \mathrm{A}$ \\
\hline Qc1 & $\begin{array}{l}3.8 \pm \\
0.7\end{array}$ & $3.3 \pm 0.6$ & $8 \pm 2$ & $163(0.25)$ & $\mathrm{N} / \mathrm{A}$ \\
\hline Qc2* $^{*}$ & $\begin{array}{l}6.6 \pm \\
0.6\end{array}$ & $5.8 \pm 0.5$ & $15 \pm 1$ & $138(0.25)$ & $113(0.26)$ \\
\hline
\end{tabular}

Ct: anthraquinone concentration after NP formation as determined by RPHPLC, Rh: mean hydrodynamic radius obtained from DLS at $90^{\circ} .{ }^{*}$ indicates concentrations after transfer in MOPS buffer. PDI - polydispersity index. N/A - not available

These peptide nanoparticles possess high stability, as storage of Qc2 loaded NPs in $\mathrm{ddH}_{2} \mathrm{O}$ at room temperature for up to 1 year did not significantly affect their size or morphology. For freshly prepared particles, the hydrodynamic radius was measured to be $159 \mathrm{~nm}$ (PDI of 0.27 ) and $173 \mathrm{~nm}$ (PDI of 0.34) after one year of storage, with TEM micrographs confirming that the spherical structure was retained (Figure S6). The very slight increase in mean hydrodynamic radius and PDI is due to the presence of few aggregates of smaller NPs, as visible in Figure S6. Similarly to the non-loaded particles, a decrease in average hydrodynamic radius from $138 \mathrm{~nm}$ (PDI of 0.25 ) to $113 \mathrm{~nm}$ (PDI of 0.26 ) was observed after transferring Qc2 loaded particles into MOPS buffer (Table 1, Figure S4 and S5). This decrease in size results in particles more suitable for extended circulation times and accumulation in tumor tissue via the EPR effect. ${ }^{[17]}$

The presence of a disulfide bond in the backbone of the amphiphilic peptide $\mathrm{H}_{3} \mathrm{SSgT}$ is intended to trigger the destruction of the NPs in reductive environments and induce the release of the anthraquinones. The release profile of the hydrophobic model payload Bodipy upon incubation in $10 \mathrm{mM}$ dithiothreitol (DTT) was previously reported and showed a rapid release of the compound, reaching $50 \%$ after 30 minutes. ${ }^{[13]}$ In the present case of anthraquinone entrapping NPs, the release profile could not be determined since the studied compounds lack fluorescent properties. However, TEM micrographs of anthraquinone loaded NPs incubated with $7 \mathrm{mM}$ of the reducing agent tris(2-carboxyethyl)phosphine (TCEP) confirmed their disassembly (Figure 2C). TCEP was selected as the preferred reducing agent due to its high stability, high reactivity at $\mathrm{pH} 6.5$ and irreversibility. ${ }^{[25,26]}$ Given that the release occurs due to the triggered decomposition of the NPs in a reductive environment, similar release profiles can be expected for various hydrophobic compounds, including the anthraquinone Qc2.

\section{Cytotoxicity of Anthraquinones upon Release from Peptide Nanoparticles}

In order to compare the cytotoxicity of our filled NPs to the free anthraquinone, we first evaluated the cytotoxicity of the anthraquinone Qc2 alone using an MTS assay on two cell lines (HEK293T and HeLa). The cells were incubated in the presence of increasing concentrations of Qc2 dissolved in ethanol. The final ethanol concentration per well, including the control cells, was $1 \%$ and did not interfere with cell viability. After $24 \mathrm{~h}$, the viability of both HEK293T and HeLa cells in presence of free Qc2 was assessed and a cytotoxic effect was observed at concentrations above $250 \mathrm{nM}$ (Figure 3).

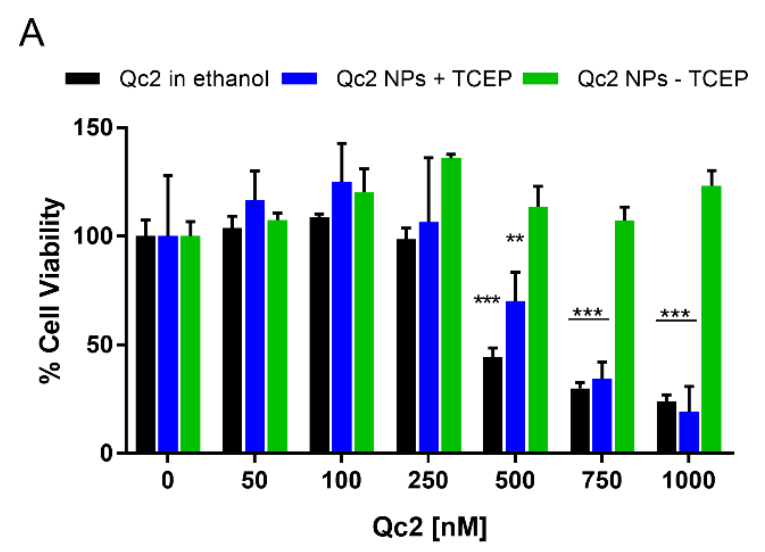

B

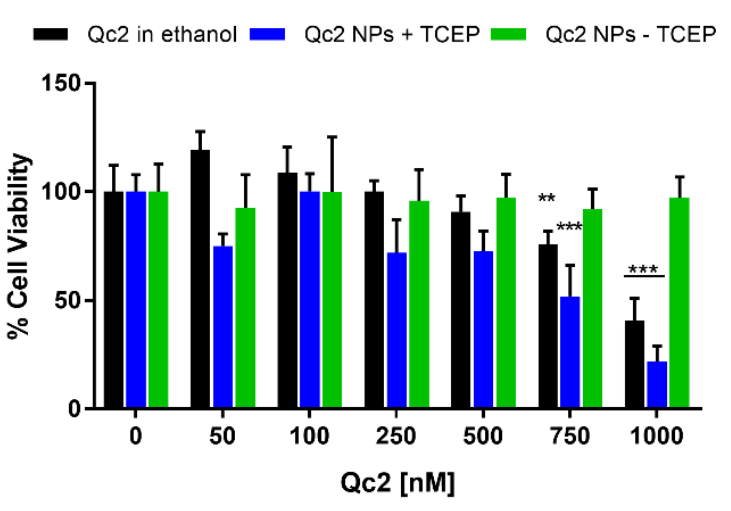

Figure 3. Viability of A) HEK293T and B) HeLa cells after exposure to the anthraquinone Qc2 (black), an equivalent concentration of Qc2 entrapped in peptide NPs with (blue) and without (green) pre-treatment with the reducing agent TCEP. The bar graphs represent the mean viability \pm SD (multiple $t-$ test, ${ }^{* *} \mathrm{P}<0.01,{ }^{* * *} \mathrm{P}<0.001, \mathrm{n}=4$ ) 
For HEK293T cells, the EC50 of Qc2 was estimated to be in the range of $500 \mathrm{nM}$ (Figure S10), whereas for HeLa cells it was higher (around $900 \mathrm{nM}$ ) (Figure S11). With respect to the HeLa cells, the EC50 is comparable to values reported for DOX.[13] Due to the higher loading efficiency of Qc2 as compared to DOX, the amount of peptide necessary to reach a similar toxicological effect is much lower for Qc2 (around 8-fold), which is a significant advantage of this drug/NP combination.

Having determined the concentration range where Qc2 elicits a toxic effect on both cell lines, we proceeded to investigate the behavior of the Qc2-loaded nanoparticles in the presence and absence of a reducing agent. In absence of a reducing agent, peptide NPs loaded with Qc2 showed no effect on either cell line (Figure 3). This confirms not only the previously reported absence of cytotoxicity for these NPs, but also efficient shielding of the tested anthraquinone through entrapment within the NPs. These results also indicate that the anthraquinone Qc2 does not leach out and is retained within the peptide NPs. For a DDS to be effective, a target-selective trigger must release the entrapped drug, preferentially. Here, once the NPs are exposed to a reductive environment, such as that surrounding tumors, ${ }^{[27]}$ the disulfide bond of $\mathrm{H}_{3} \mathrm{SSgT}$ breaks and the NPs are destroyed, leading to drug release. To confirm that the NPs can efficiently release their payload in reductive environments, we incubated the cells in presence of NPs pre-treated with $7 \mathrm{mM}$ of the reducing agent TCEP. We observed a significant decrease in cell viability, for both cell lines, in the presence of the pre-treated NPs, reaching levels comparable to those of the free anthraquinone (Figure 3 , S10 and S11). This proves that the NPs release the active anthraquinone Qc2 in a reductive environment. TCEP was used to ensure the full disassembly of the NPs and to study the release of Qc2; however, it would be interesting to further study the release of Qc2 in a 3D cell culture where the tumor microenvironment can be accurately modeled and the use of external reducing agents would not be necessary. ${ }^{[28,29]}$ In addition, the MCM structure of the NPS is expected to hinder the accessibility of the disulfide bonds to enzymes present in biological fluids, thus limiting their enzymatic degradation. Preliminary results suggest that $24 \mathrm{~h}$ incubation in fetal calf serum does not significantly affect the morphology of the NPs (Figure S12).

\section{Conclusions}

Three different anticancer drug candidates, based on redox active anthraquinones, were entrapped within peptide-based NPs of approximately $200 \mathrm{~nm}$. The NPs were formed by self-assembly of a reduction responsive amphiphilic peptide. One compound, Qc2, previously shown to produce ROS at a higher rate than DOX, was loaded within NPs with a high entrapment efficiency, reaching up to 15 mg per $\mathrm{g}$ peptide $(5.8 \mathrm{~mol} \%)$. This is more than 8 times higher than the loading efficiency previously reported for DOX. The compound Qc2 exhibited significant toxicity towrds both HEK293T and HeLa cells, whereas NPs loaded with Qc2 showed no cytotoxicity, at comparable anthraquinone concentrations. Pre-treatment of Qc2 loaded NPs with reducing agent resulted in release of Qc2 and an increase in cytotoxicity to levels comparable to the free compound. The efficient loading of biocompatible peptide based NPs with hydrophobic anthraquinones, combined with the stimuli-responsive release of the payload, confirms the potential of this anthraquinonepeptide system as a drug delivery vehicle for anti-cancer drug candidates. Indeed, a NP based delivery system capable of targeting tumor sites through the EPR effect and capable of releasing highly toxic molecules selectively, should allow for efficient treatment while minimizing the side effects caused by non-selective drugs. While this system shows promising results, further characterization in vivo is needed to fully assess its clinical potential.

\section{Experimental Section}

\section{Materials}

The peptide $\mathrm{H}_{3} \mathrm{SSgT}$ with sequence $\mathrm{H}_{2} \mathrm{~N}-\mathrm{H} 3-\mathrm{X}-[\mathrm{W}-\mathrm{D} \mathrm{L}]_{3}-\mathrm{W}-\mathrm{CONH}_{2}$, (with $\mathrm{X}=-\left(\mathrm{CH}_{2}\right)_{2}-\mathrm{S}-\mathrm{S}-\left(\mathrm{CH}_{2}\right)_{2}-\mathrm{NH}-\mathrm{CO}-\left(\mathrm{CH}_{2}\right)_{2}-\mathrm{CO}-$ and $\mathrm{DL}=\mathrm{D}$ Leucine) was synthesized, purified and characterized as previously described. ${ }^{[1]}$ The anthraquinones Qc1 (2-phenyl-4-(butylamino)naphtho[2,3$h$ ]quinoline-7,12-dione), Qc2 (2-phenyl-4-(octylamino)naphtho[2,3- $h$ ]quinoline7,12-dione) and Qn (2-phenyl-5-nitronaphtho[2,3-g]indole- 6,11-dione) were synthesized and purified as described previously. ${ }^{[23]}$

\section{General Methods}

Methods on peptide nanoparticles formation, characterization and anthraquinone quantification can be found in the SI.

\section{Payload Embedding in Peptide Nanoparticles}

The anthraquinones were dissolved in ethanol to a concentration of $100 \mu \mathrm{M}$ and stored in the dark. Typically, solutions with final concentrations of $0.2 \mathrm{mg} \mathrm{mL}^{-1}$ peptide and $12 \mu \mathrm{M}$ anthraquinone were prepared in $50 \%$ ethanol and dialyzed as described in the SI. The final anthraquinone concentration was determined by RP-HPLC

\section{Anthraquinone Quantification}

Anthraquinone concentration was determined by RP-HPLC (Prominence 20A, Shimadzu, Japan) using a C18 reverse phase column (Merck Chromolith RP-18e, 100x4.6 mm, Merck, Germany) and a gradient from $0-100 \%$ acetonitrile/water (0.1\% TFA) over 10 minutes. Injection volumes were $30 \mu \mathrm{L}$ and the chromatograms were recorded at $280 \mathrm{~nm}$. Concentrations were calculated using a calibration curve obtained by reporting the area under the peak as a function of the anthraquinone concentration. The calibration samples were prepared in triplicate by dilution of a stock solution of the anthraquinone in ethanol. Peptide containing samples were dissolved in 1:1 with dimethylformamide and vortexed for $30 \mathrm{~s}$ prior to injection.

\section{Cell Culture}

HEK293T and HeLa cells were cultured in Dulbecco's Modified Eagle Medium with GlutaMAX ${ }^{\mathrm{TM}}-\mathrm{I}$ and supplemented with $10 \%$ Fetal calf serum (FCS) and $1 \%$ penicillin/Streptomycin (100 units $\mathrm{mL}^{-1}$ penicillin 
and $100 \mu \mathrm{g} \mathrm{mL} \mathrm{m}^{-1}$ Streptomycin). Cells were kept in a humidified atmosphere with $5 \% \mathrm{CO}_{2}$ at $37^{\circ} \mathrm{C}$.

\section{Cell Viability Assay}

To determine cell viability, the CellTiter $96 \AA$ AQueous One Solution Cell Proliferation Assay (MTS) was used according to the manufacturer's protocol. Cells were seeded at a density of 5000 cells/well in a 96-well plate and cultured for $24 \mathrm{~h}$. After $24 \mathrm{~h}$, free Qc2 in ethanol, NPs containing Qc2 in MOPS buffer, or NPs containing Qc2 pre-incubated $\left(4 \mathrm{~h}\right.$ at $37^{\circ} \mathrm{C}$ ) with $7 \mathrm{mM}$ TCEP in MOPS buffer were added to the cells to reach final concentrations of Qc2 ranging from 0 to $1 \mathrm{mM}$. Concentrations of ethanol or TCEP were normalized for all tested Qc2 concentrations to $1 \mathrm{vol} \%$ and $1 \mathrm{mM}$ respectively. Cells were further incubated in presence of compounds for $24 \mathrm{~h}$ after which $20 \mu \mathrm{L}$ MTS reagent was added to each well. After $2 \mathrm{~h}$ absorbance was measured at $490 \mathrm{~nm}$ using a Spectramax plate reader (Molecular Devices LLC, USA). Background absorbance, measured in control wells containing all assay components except cells, was subtracted from each well and normalized to control cells containing all components except Qc2 or Qc2 loaded NPs. The normalized data was plotted using Prism7 (GraphPad Software) and statistics were performed using the built-in multiple t-test function $(P<0.01$ was considered significant, $n=4$ ).

\section{Supplementary Material}

Supporting information for this article is available on the WWW under http://dx.doi.org/10.1002/MS-number.

\section{Acknowledgements}

The authors would like to acknowledge the generous financial support from the Swiss National Science Foundation (SNSF), particularly in light of the National Center for Competence in Research for Molecular Systems Engineering (NCCR-MSE) and the University of Basel. The authors would also like to thank Dr. Severin J. Sigg for providing the peptide and for fruitful discussions, Evi Bieler for SEM imaging and Prof. L. Fedenok for the synthesis of the anthraquinone derivatives. P.

U. R, would like to thank Freiwillige Akademische Gesellschaft Basel for financial support.

\section{Author Contribution Statement}

P.U.R., I.C., J.G, L.W. and C.G.P. designed the studies. P.U.R. and

I.C. performed the experiments and all listed authors wrote/edited the manuscript. The authors declare no competing financial interest.

\section{References}

[1] O. Tacar, P. Sriamornsak, C. R. Dass, 'Doxorubicin: an update on anticancer molecular action, toxicity and novel drug delivery systems', J. Pharm. Pharmacol. 2013, 65 157-170

[2] J. V. McGowan, R. Chung, A. Maulik, I. Piotrowska, J. M. Walker, D. M. Yellon, 'Anthracycline Chemotherapy and Cardiotoxicity', Cardiovasc Drugs Ther 2017, 31, 63-75.
[3] N. Erathodiyil, J. Y. Ying, 'Functionalization of Inorganic Nanoparticles for Bioimaging Applications', Acc. Chem. Res. 2011, 44, 925-935.

[4] Y. H. Tsou, X. Q. Zhang, H. Zhu, S. Syed, X. Y. Xu, 'Drug Delivery to the Brain across the Blood-Brain Barrier Using Nanomaterials', Small 2017, 13

[5] J. L. Shen, W. Zhang, R. G. Qi, Z. W. Mao, H. F. Shen, 'Engineering functional inorganic-organic hybrid systems: advances in siRNA therapeutics', Chem. Soc. Rev. 2018, 47, 1969-1995

[6] U. Kanwal, N. Irfan Bukhari, M. Ovais, N. Abass, K Hussain, A. Raza, 'Advances in nano-delivery systems for doxorubicin: an updated insight', J Drug Target 2018, 26 296-310.

[7] G. Gunkel-Grabole, S. Sigg, M. Lomora, S. Lorcher, C. G. Palivan, W. P. Meier, 'Polymeric 3D nano-architectures for transport and delivery of therapeutically relevant biomacromolecules', Biomater Sci 2015, 3, 25-40.

[8] L. Messager, J. Gaitzsch, L. Chierico, G. Battaglia, 'Novel aspects of encapsulation and delivery using polymersomes', Curr Opin Pharmacol 2014, 18, 104-111.

[9] P. U. Richard, J. T. Duskey, S. Stolarov, M. Spulber, C. G. Palivan, 'New concepts to fight oxidative stress: nanosized three-dimensional supramolecular antioxidant assemblies', Expert Opin Drug Deliv 2015, 12, 1527-1545.

[10] Y. Matsumura, H. Maeda, 'A New Concept for Macromolecular Therapeutics in Cancer Chemotherapy: Mechanism of Tumoritropic Accumulation of Proteins and the Antitumor Agent Smancs', Cancer Res. 1986, 46, 6387.

[11] V. Mikhalevich, I. Craciun, M. Kyropoulou, C. G. Palivan, W. Meier, 'Amphiphilic Peptide Self-Assembly: Expansion to Hybrid Materials', Biomacromolecules 2017, 18, 34713480.

[12] D. de Bruyn Ouboter, T. B. Schuster, A. Mantion, W. Meier, 'Hierarchical Organization of Purely Peptidic Amphiphiles into Peptide Beads', The Journal of Physical Chemistry C 2011, 115, 14583-14590.

[13] S. J. Sigg, V. Postupalenko, J. T. Duskey, C. G. Palivan, W. Meier, 'Stimuli-Responsive Codelivery of Oligonucleotides and Drugs by Self-Assembled Peptide Nanoparticles', Biomacromolecules 2016, 17, 935-945.

[14] S. J. Sigg, T. B. Schuster, W. P. Meier, 'Self-assembled structures from amphiphilic peptides', Chimia (Aarau) 2013, 67, 881-884

[15] N. Rahoui, B. Jiang, N. Taloub, Y. D. Huang, 'Spatiotemporal control strategy of drug delivery systems based nano structures', J Control Release 2017, 255, 176-201.

[16] M. van Elk, B. P. Murphy, T. Eufrasio-da-Silva, D. P. O'Reilly, T. Vermonden, W. E. Hennink, G. P. Duffy, E. Ruiz-Hernandez, 'Nanomedicines for advanced cancer treatments: Transitioning towards responsive systems', Int. J. Pharm. 2016, 515, 132-164.

[17] S. D. Steichen, M. Caldorera-Moore, N. A. Peppas, 'A review of current nanoparticle and targeting moieties for the delivery of cancer therapeutics', Eur. J. Pharm. Sci. 2013, 48, 416-427.

[18] P. C. t. I. Balaure, D. Gudovan, I. A. Gudovan, 'Smart triggered release in controlled drug delivery', Curr. Drug Targets 2016, 17

[19] S. Mura, J. Nicolas, P. Couvreur, 'Stimuli-responsive nanocarriers for drug delivery', Nat. Mater. 2013, 12, 9911003.

[20] R. Cheng, F. Feng, F. Meng, C. Deng, J. Feijen, Z. Zhong, 'Glutathione-responsive nano-vehicles as a promising platform for targeted intracellular drug and gene delivery', $J$ Control Release 2011, 152, 2-12.

[21] N. Polyakov, T. Leshina, L. Fedenok, I. Slepneva, I. Kirilyuk, J. Furso, M. Olchawa, T. Sarna, M. Elas, I. Bilkis, L. Weiner, 'Redox-Active Quinone Chelators: Properties, Mechanisms of Action, Cell Delivery, and Cell Toxicity', Antioxid Redox Signal 2018, 28, 1394-1403.

[22] L. M. Weiner, in Methods Enzymol., Vol. 233, Academic Press, 1994, pp. 92-105.

[23] S. I. Dikalov, G. V. Rumyantseva, A. V. Piskunov, L. M Weiner, 'Role of quinone-iron(III) interaction in NADPHdependent enzymic generation of hydroxyl radicals', Biochemistry 1992, 31, 8947-8953. 
[24] R. Pinkus, L. M. Weiner, V. Daniel, 'Role of Quinone-

Mediated Generation of Hydroxyl Radicals in the Induction of Glutathione S-Transferase Gene Expression',

Biochemistry 1995, 34, 81-88.

[25] J. C. Han, G. Y. Han, 'A Procedure for Quantitative

Determination of Tris(2-Carboxyethyl)phosphine, an

Odorless Reducing Agent More Stable and Effective Than

Dithiothreitol', Anal. Biochem. 1994, 220, 5-10.

[26] O. Dmitrenko, C. Thorpe, R. D. Bach, 'Mechanism of SN2 Disulfide Bond Cleavage by Phosphorus Nucleophiles. Implications for Biochemical Disulfide Reducing Agents', The Journal of Organic Chemistry 2007, 72, 8298-8307.

[27] K. V. V., G. R. J., 'Janus-Faced Tumor Microenvironment and Redox', Antioxid. Redox Signaling 2014, 21, 723-729.

[28] R. Maddaly, P. V., K. S.R., A. E., S. F. D. Paul, '3D Cell Culture Systems: Advantages and Applications', J. Cell. Physiol. 2015, 230, 16-26.

[29] S. A. Langhans, 'Three-Dimensional in Vitro Cell Culture Models in Drug Discovery and Drug Repositioning',

Frontiers in Pharmacology 2018, 9. 